\title{
Supranuclear Ophthalmoplegia in Powassan Encephalitis
}

\author{
Pascale Trépanier, Vilayvong Loungnarath, Alain Gourdeau, Christiane Claessens, Martin Savard
}

Can. J. Neurol. Sci. 2010; 37: 890-892

Powassan encephalitis (PE) is a rare tick-borne encephalitis. ${ }^{1}$ Since the first diagnosed case in 1958 in Powassan, ${ }^{2}$ Ontario, Canada, only 36 cases have been reported. ${ }^{3}$ We describe herein a case of PE with acute supranuclear ophthalmoplegia (SO), an unusual clinical feature which, to our knowledge, has only been reported once. ${ }^{4}$

\section{CASE}

In November 2008, a 61-year-old man from Quebec City, Canada, presented with a three-day fever and an acute confusional state. He was disorientated with incoherent speech, complaining of dizziness and headache. His wife had noticed that he deviated towards the left side while walking. A month earlier, he went hunting near Victoriaville, about 100 kilometers southwest of Quebec City. No rash or bite marks were noted. He had no other recent travel. Past medical history was noncontributory.

Physical examination revealed a somnolent but easily arousable patient. Vitals signs were within normal limits except for a rectal temperature of $41^{\circ} \mathrm{C}$. Examination of the neck revealed no stiffness. Extraocular movements were initially slightly limited on the left side with a suspicion of a left VIth nerve dysfunction with no evidence of VIIth nerve palsy, but cranial nerves examination was limited due to lack of cooperation. Fundus examination did not show papilledema. The remaining neurological and physical exams were within normal limits, but gait could not be properly assessed.

White blood cell count was $13.60 \times 10^{9} / \mathrm{L}$ (normal 4.0-10.0 x $\left.10^{9} / \mathrm{L}\right)$. The cerebrospinal fluid was noticeable for $65 \times 10^{6}$ white blood cells/L (55 mononuclears, 9 polynuclears, 1 other) and elevated protein at $1.06 \mathrm{~g} / \mathrm{L}$ (normal $0.15-0.40 \mathrm{~g} / \mathrm{L}$ ).

Head CT was normal. Initial MRI with and without contrast enhancement showed a mild FLAIR/T2 hyperintensity at the junction between the internal capsule and the caudate nucleus on the left side, with also some involvement of the junction between putamen and external capsule (Figure, top). No brainstem abnormality was noticed. Standard EEG revealed a light slow, diffuse background dysrythmia. There was no periodic lateralized epileptiform discharge, spike or sharp wave. Patient was then started on a large specter antibiotic and antiviral regimen covering most encephalitis and meningitis, including zoonosis.

On the following days, the patient presented some agitation and tremors. High fever persisted for four days despite treatment. Neuro-ophthalmologic consultant noticed a complete conjugate vertical ophthalmoplegia for smooth pursuit and saccades. Horizontally, a 50\% limitation of smooth pursuit was noticed on the left while it was normal on the right, associated with saccade slowing in both directions. An abnormal optokinetic reflex in all directions was also noted. The oculovestibular reflex (done by oculocephalic stimulation) was normal in all directions, which confirmed SO. Visual acuity was not impaired.

The MRI was repeated five days later and did show complete resolution of the previous mild hyperintensity (Figure, bottom). Lumbar puncture was also repeated and showed a reduction of white blood cells $\left(21 \times 10^{6}\right.$ white blood cells/L) and proteins (0.86 g/L).

Microbiological tests on cerebrospinal fluid were negative including polymerase chain reaction analyses for Herpes simplex virus, Varicella zoster virus, Ebstein-Barr virus, cytomegalovirus, adenovirus, enterovirus and Tropheryma whipplei, this last one being also ruled out by a duodenal biopsy. Numerous serologies were done on early and late serum, including those for West Nile virus, Lyme disease and tularemia. Antibodies to Powassan virus were detected in inhibition hemagglutination assay with specific antigen. Infection was confirmed at the National Microbiology Laboratory by plaque reduction neutralization testing with a titer of 40 on early serum and $\geq 640$ on late serum.

Two months later, the patient had almost entirely recovered and did not complain of residual symptoms, except for a persisting difficulty with down gaze. His coordination and balance were almost normal. A year and a half later, down gaze was still affected with a $30 \%$ limitation of smooth pursuit downward combined with a near complete abolition of saccades in the same direction and a light decrease of the optokinetic reflex in all directions, oculovestibular reflex still being preserved (see Video on-line). Retrospectively, our patient did recall a tick bite while he was hunting.
From the Département de microbiologie médicale et de maladies infectieuses (PT, VL), Département d'ophtalmologie (AG), Département des sciences neurologiques (MS), Centre Hospitalier Affilié Universitaire de Québec, Hôpital de l'Enfant-Jésus,

Université Laval, Québec City, Québec, Canada, and from Laboratoire de santé publique de l'Institut national de santé publique du Québec (CC).

Received March 29, 2010. Final Revisions Submitted May 25, 2010. Correspondence to: Martin Savard, Centre Hospitalier Affilié Universitaire de Québec, Hôpital de l'Enfant-Jésus, 1401, 18e Rue, Québec, Québec, G1J 1Z4, Canada. 


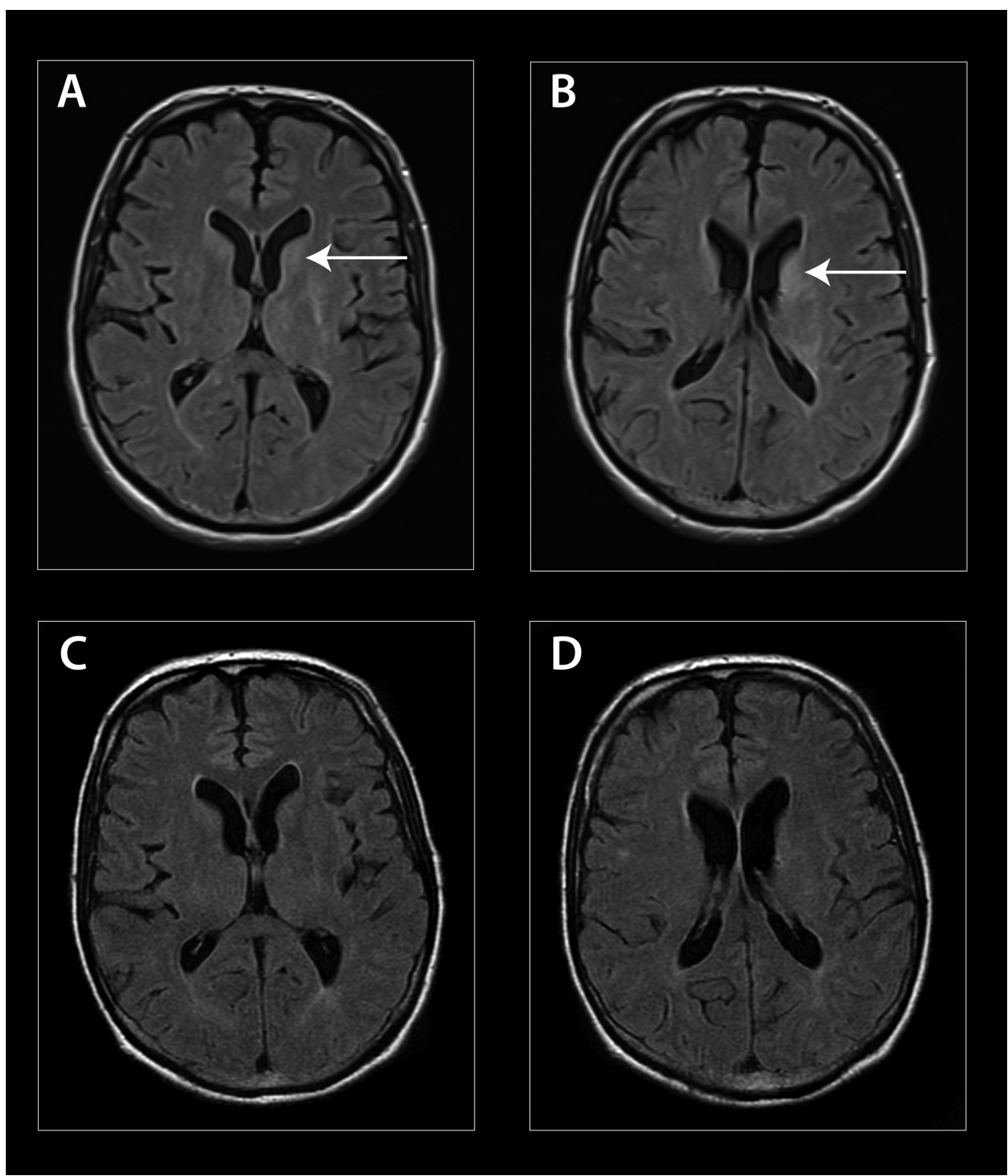

Figure: A and B: FLAIR sequence on MRI, on November 30, 2008, showing mild hyperintensity at the left internal capsule/caudate nucleus junction (arrow) and also at the putamen and external capsule junction. $C$ and D: Same sequence, five days later, showing resolution of this subcortical hyperintensity.

\section{DisCUSSION}

Powassan virus is a flavivirus found in Europe, northeastern United States, and eastern Canada. ${ }^{5}$ From 1958 to 1998,27 cases have been reported. From 1999-2006, a peak was noted with ten cases in the United States and one in Canada. ${ }^{3,6}$ Two cases were also reported in Quebec in 2008-2009. ${ }^{6}$ This rise has been linked to the introduction of West Nile virus in North America and a concomitant Powassan infection detection through increased testing. ${ }^{7}$ Fatality is around $15 \%$ and about two thirds of patients have significant neurologic sequelae. ${ }^{5}$ It is linked to four tick species (Ixodes cookei, Ixodes marxi, Ixodes spinipalpus, and Dermacentor andersoni) with small to medium-size mammals for hosts. ${ }^{5}$ Most patients do not recall tick bites, probably because of its very small size. ${ }^{1}$ Most infections occur between June and September but cases have been reported outside that range. The incubation period ranges from 8 to 34 days. ${ }^{1}$ Our patient went hunting at the end of October, and symptoms began four weeks later.

The classic presentation generally includes fever, weakness, somnolence, gastrointestinal complaints, headache and confusion. ${ }^{1,3,5}$ The combination of ophthalmoplegia and preserved oculovestibular reflex in our patient is consistent with $\mathrm{SO}$, which is very unusual in an infectious acute setting and only reported to occur with Whipple disease, ${ }^{8}$ disseminated histoplasmosis ${ }^{9}$ and postencephalitic parkinsonism ${ }^{10}$ among other infections. However, in our patient, Whipple disease has been ruled out by duodenal biopsy, disseminated histoplasmosis 
was not considered mainly for epidemiological reasons, and postencephalitic parkinsonism was not considered since SO is a late phenomenon with this disease, oculogyric crisis being seen in the acute encephalitic phase instead of ophthalmoplegia. Finally, acute SO can happen with paraneoplastic limbic encephalitis, ${ }^{11}$ which could give the lumbar puncture profile we have seen in this patient, but we did not consider that diagnosis since the patient was clearly febrile.

Some doubts remain as to the exact pathogenesis of SO in PE, which should be either by direct infection of the relevant brain regions or by an auto-immune process by molecular mimicry; however, previous pathologic report documented the presence of the virus in the central nervous system. ${ }^{12}$ The lesion found in our patient MRI (Figure) does not seem to be relevant to this clinical finding. We could hypothesize that a mesencephalic lesion may have created that clinical picture, since both reflexive and intentional saccades were affected, with a particular involvement of the superior colliculus, but this may not explain the initial smooth pursuit impairment, more involved at the pontine level among the brainstem, which consequently necessitate multifocal brainstem lesions. ${ }^{13}$ Multifocal frontal and parietal cortical lesions could have been evoked when the patient initially consulted, but the later evolution to a mainly vertical involvement of saccades argues against it, in favour of the mesencephalic theory. Moreover, an autopsy done previously on a patient with $\mathrm{PE}$ but without SO showed prominent involvement of large neurons in the brainstem, cerebellum, basal ganglia, thalamus, and spinal cord, but not in the cortex. ${ }^{12}$

To our knowledge, this is the second reported case of SO in PE. ${ }^{4}$ Furthermore, it suggests that, when confronted with a case of SO and encephalitis, it may be relevant to include PE in the differential diagnosis, particularly when the epidemiology is suggestive.

\section{REFERENCES}

1. Gholam BIA, Puksa S, Provis JP. Powassan encephalitis: a case report with neuropathology and literature review. Can Med Assoc J. 1999;61(11):1419-22.

2. McLean DM, Donahue WL. Powassan virus: isolation of virus from a fatal case of encephalitis. Can Med Assoc J. 1959;80(9): 708-11.

3. Hinten SR, Beckett GA, Gensheimer KF, et al. Increased recognition of Powassan encephalitis in the United States 19992005. Vector Borne Zoonotic Dis. 2008;8(6):733-40.

4. Lessell S, Collins, TE. Ophthalmoplegia in Powassan encephalitis. Neurology. 2003;60(10):1726-27.

5. Romero JR, Simonsen KA. Powassan encephalitis and Colorado tick fever. Infect Dis Clin North Am. 2008;22(3):545-59.

6. Institut national de santé publique du Québec. STATLABO. 2009 Oct [cited 2010 Feb 23];8(10);5. French. Available from: http://www.inspq.qc.ca/bulletin/STATLABO/default.asp?E=p.

7. CDC. Outbreak of Powassan encephalitis - Maine and Vermont, 1999-2001. MMWR Morb Mortal Wkly Rep. 2001;50(35); 761-4.

8. Schneider T, Moos V, Loddenkemper C, Marth T, Fenollar F, Raoult D. Whipple's disease: new aspects of pathogenesis and treatment. Lancet Infect Dis. 2008;8(3):179-90.

9. Perry JD, Girkin CA, Miller NR, Mann RB. Disseminated histoplasmosis causing reversible gaze palsy and optic neuropathy. J Neuroophthalmol. 1999;19:40-3.

10. Wenning GK, Jellinger K, Litvan I. Supranuclear gaze palsy and eyelid apraxia in postencephalitic parkinsonism. J Neural Transm. 1997;104:845-65.

11. Senties-Madrid H, Vega-Boada F. Paraneoplastic syndromes associated with anti-Hu antibodies. Isr Med Assoc J. 2001;3(2): 94-103.

12. Tavakoli NP, Wang H, Dupuis M, et al. Fatal case of deer tick virus encephalitis. N Engl J Med. 2009;360(20):2099-107.

13. Lee AG, Brazis PW. Clinical pathways in neuro-ophthalmology: an evidence-based approach. New York, New York: Thieme; 1998. 